colonoscopies performed. In those with newly diagnosed IBD at colonoscopy confirmed on histology, clinical data including demographics, disease characteristics, treatment and outcome were obtained from case note and electronic patient record review.

Results Of 378424 patients invited, 172244 were screened, representing an uptake of $45.52 \%$ and FOBt positivity of $2.71 \%$. Colonoscopy was performed in 4195 patients (female 1761). Polyps were detected in 1870 (40.14\%), cancer in 279 (5.99\%) and 1216 (26.10\%) had a normal examination. 83 patients had endoscopic appearance suggestive of IBD, confirmed at histology in 44 . Seven patients were excluded as the diagnosis of colitis preceded the screening examination on case note review. Eleven of 37 incidental cases were female. Median age at diagnosis was 64. Twelve patients had Crohn's disease (CD), 22 ulcerative colitis (UC) and three had IBDtype unclassified (IBDU). 31 patients had follow-up data available with a mean follow-up period of 24.4 months. Fifteen patients $(48.4 \%)$ were asymptomatic at diagnosis. Mean values for CRP were 11.8, $\mathrm{Hb}$ 13.8, Platelets 278.5, and Albumin 42.9. Treatment included steroids (8), 5-ASA (25), immunomodulators (azathioprine 5; methotrexate 1) and anti-TNF (infliximab 2; adalimumab 1). None required surgery. Those requiring immunomodulators and/or antiTNF therapy (male 4; female 1) had asymptomatic extensive UC, symptomatic left sided UC, symptomatic left-sided IBDU, symptomatic Crohn's colitis and symptomatic stricturing terminal ileal $\mathrm{CD}$ at diagnosis.

Conclusion An incidental diagnosis of IBD is not uncommon and with the advent of bowel cancer screening this number is set to increase. These patients may present an important model for study of early disease with novel insights and evolving treatment paradigms.

Competing interests None declared.

\section{PWE-241 VITAMIN D STATUS IN INFLAMMATORY BOWEL DISEASE: ARE CLINICIANS SEEING THE LIGHT?}

doi:10.1136/gutjnl-2012-302514d.241

R 0 Butcher, ${ }^{*}$ L E Loo, E Nixon, X McFarlane, J K Limdi. Department of Gastroenterology, Pennine Acute Hospitals, Manchester, UK

Introduction There has been resurgent interest in recent years in the pro-hormone vitamin $\mathrm{D}$ beyond its classical role in bone metabolism recognising its plausible effects in immune regulation. The aim of our study was to review practice relating to vitamin $\mathrm{D}$ assessment among IBD patients.

Methods We conducted a retrospective review of 300 consecutive patients attending IBD clinics at our hospital. Clinical data including demographics, disease characteristics and therapy were obtained from case note and electronic patient record review. Measurement of serum 25-hydroxyvitamin D (25-OHD) concentration subsequent to IBD diagnosis was noted.

Results Of 300 IBD patients reviewed 141 were female. The median age was 47.5 (range 16-91) and mean disease duration 9.6 years. 137 patients had Crohn's disease, 152 patients ulcerative colitis and 11 patients had IBD-type unclassified (IBDU). 126 (42.0\%) patients were current or ex-smokers. Vitamin D status was assessed in 45 $(15.0 \%)$ patients. In 41 patients $(91.1 \%)$ measurement was undertaken within the last 2 years. The mean and median serum $25-\mathrm{OHD}$ level was 19.1 and $17.0 \mathrm{ng} / \mathrm{ml}$ respectively (range 5-49.9). Nine $(20.0 \%)$ of these patients had levels $<10 \mathrm{ng} / \mathrm{ml}$ consistent with deficiency and 17 (37.8\%) levels $<20 \mathrm{ng} / \mathrm{ml}$. Of the nine patients with vitamin $\mathrm{D}$ deficiency (three males; six females), six had Crohn's disease and three had ulcerative colitis. Of the Crohn's patients, three had ileo-colonic, two colonic and one ileal disease. Three had non-stricturing and non-penetrating disease, two stricturing and one penetrating disease. Of the ulcerative colitis patients one had extensive disease, one left-sided disease and one had proc- titis. All patients had received steroids during the course of their disease and three patients received azathioprine, four anti-TNF (three infliximab; one adalimumab) and four had previous surgery. Patients with vitamin $\mathrm{D}$ deficiency had significant disease requiring immunomodulator, anti-TNF therapy and surgery in this cohort.

Conclusion Vitamin D assessment in IBD patients is suboptimal. Hypovitaminosis D is under-recognised and consequently undertreated. The myriad emerging roles of vitamin $\mathrm{D}$ in the pathogenesis of IBD emphasise the importance of recognition and optimisation of vitamin $\mathrm{D}$ status to above $30 \mathrm{ng} / \mathrm{ml}$ in this patient group.

Competing interests None declared.

\section{PWE-242 ACCESSING INFORMATION ON IBD: A SURVEY OF SOURCES AND RELIABILITY OF INTERNET RESOURCES}

doi:10.1136/gutjnl-2012-302514d.242

R A Ley Greaves, * L F Wilkinson, T Orchard. Imperial College, London, UK

Introduction Crohn's and Ulcerative Colitis (UC) are chronic diseases, often requiring lifelong treatment. They can be variable therefore accurate and useful patient information is vital. With an increasing number of sources this can be a cause of confusion for patients. As part of a service evaluation this study examined the information given to patients by doctors, other sources used, how they chose their information and their accuracy.

Methods 100/113 patients agreed to answer a short survey. Topics were: Sources of information, internet usage, checks made on information and reasons for choices. Separately an internet search was performed with three major search engines using five search terms (IBD, UC, Crohn's, Ulcerative colitis and inflammatory bowel disease). From the top 10 hits on each 22 websites that were relevant to the search term were then checked against 5 criteria for accreditation of information.

Results Of 100 patients (43 male, 57 female) with an average age of 44 (18-81). There were 47 Crohn's and 53 UC patients. $65 \%$ had suffered for over 5 years. $48 \%$ wanted more information than given in clinic and $91 \%$ had sought further information. Of those who sought further information $90 \%$ used the internet, $55 \%$ patient leaflets and $25 \%$ used books. Sources used varied with age, $78 \%$ of over 65 s didn't use the internet while only $6 \%$ of under 30 s doing research didn't. Leaflet use in the under 30s was $48 \%$ while being $78 \%$ in the over $65 \mathrm{~s}$. Using the search terms in Google, Yahoo and bing an average of 1, 1.5 and 2 sponsored advertising links were highest ranked respectively. $67 \%$ of those using the internet picked websites on their search engine ranking with $20 \%$ picking on recommendation. $40 \%$ made no checks on the information they found whereas $23 \%$ said they "read around". Of the 22 relevant websites 7 filled 5 accreditation criteria and 6 scored 2 or less. Crohn's and Colitis UK (NACC) website scored highly for accreditation and $45 \%$ of patients used it $62 \%$ of whom had been recommended it by their doctor. However its mean search engine ranking was 7 th and it only appeared in the top ten in 9/12 searches. In the free text section a number of patients commented on concerns about inaccurate information.

Conclusion The results show patient information is mainly sourced from the internet and patient leaflets. Internet information is of varying quality and search engine rankings are influenced by sponsored links directed at IBD patients as opposed to accredited sites. Data on use of the NACC website with and without recommendation show simple measures such as a health care professional taking time to advise a patient may direct them to towards better information and away from lower quality potentially misleading sites. Many patients commented that websites with unsubstantiated information caused them significant concern.

Competing interests None declared. 